\title{
Rare impaling injury to the neck
}

Appuhamy P*, Perera J, Fernando R

Department of Forensic Medicine and Toxicology, Faculty of Medicine, Colombo

*Corresponding author - Tel: 0718255372, e mail: prasannaappuhamy@yahoo.com

MLJSL.Vol 3. No 1. March. pp 29-32

\begin{abstract}
Introduction

Impaling injuries are uncommon. They result mostly when rigid objects with a pointed or tapering end penetrate the body. Circumstances include falls and traffic accidents. Individuals falling or jumping from a structure onto pointed objects such as a spiked fences or pointing metal rods are common scenarios. Current case is a death of a heavy alcoholic falling onto a pointed stump of a tree injuring the neck.
\end{abstract}

\section{History and scene examination}

A 65 year old male had left home to buy an alcoholic drink, and was found fallen in prone position the following day, in an area with a lot of bushes. The injury over the left side of the neck was in contact with a pointed blood stained stump of a tree about $45 \mathrm{~cm}$ in height.

\section{Autopsy}

There was a V shaped defect of the shirt front just below the collar. The shirt was thickly stained with blood around the defect. There was a gaping elliptical shaped penetrating wound over the front of left lower neck with irregular and abraded margins. Underlying soft tissue and muscle contusion were present. There was complete transection of the internal jugular vein. The injury had penetrated into the thoracic cavity causing a $400 \mathrm{ml}$ haemothorax. There were no injuries in the rest of the body. All organs were pale.

\section{Conclusion}

A rare case of impaling injury is reported. In similar cases where the body of the victim is discovered further away from the tree stump, foul play could be suspected. If the tree stump was missed at the crime scene this suspicion would be further strengthened with the possibility of miscarriage of justice. This case illustrates the importance of a thorough examination of the scene to detect the cause of impaling injuries.

Key words: Impalement, impaling, neck injuries, penetrating, tree stump, scene examination 


\section{Introduction}

Impalement or impaling injuries are uncommon and occurs when a portion of the body is penetrated by a rigid object with a pointed or tapering end. The objects frequently remain in the wound. They are usually caused in falls and traffic accidents. Individuals fall or jump from a structure onto a pointed object such as a fence [01], or a pointing metal rod. Impaling on unguarded protruding steel reinforcing bars (rebars) is a common cause of accident at construction sites [02]. Impalement on a golf club shaft also has been reported [03]. In traffic accidents, a vehicle might crash onto an obstacle e.g. a fence, which in part or whole is driven into the car, impaling the driver or a passenger. Current case is a death of a heavy alcoholic following a fall onto an irregularly cut tree stump impaling the neck.

\section{Case report}

\section{History and scene examination}

A 65 year old male had left home to buy an alcoholic drink and was found fallen the following day in prone position on the ground in an area with a lot of bushes (Figure 01). When the body was moved there was a thickly blood stained stump of a tree measuring $45 \mathrm{~cm}$ in length. It was sharply but incompletely cut and had a protruding part with a tapering end (Figure 02). This protruding part was in contact with a defect in the shirt and underlying injury over the neck. There was a large blood pool on the ground close to the stump. At the undisturbed scene there was an unopened bottle of arrack.

\section{Autopsy}

There was a $V$ shaped defect just below the collar on the front of the shirt left side (Figure 03). Each limb of "V" measured $6 \mathrm{~cm}$. Left upper half of the shirt was stained with blood and was thickly stained around the defect. There was a gaping elliptical shaped penetrating wound over the front of left lower neck with irregular and abraded margins (Figure 04).
Figure 01. Victim found impaled on a stump of a tree

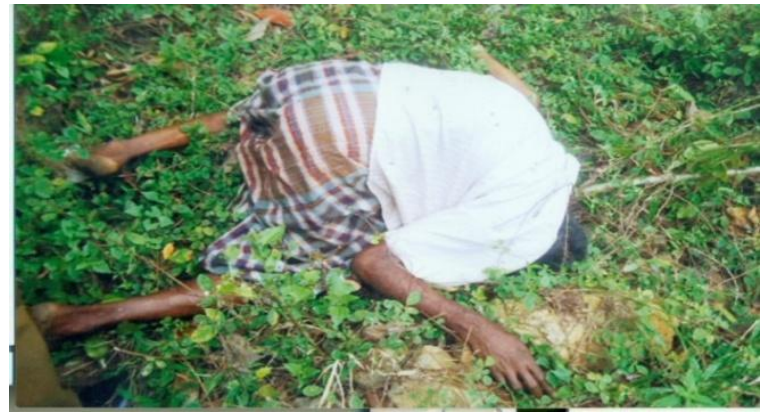

Figure 02. Stump of tree with tapering end

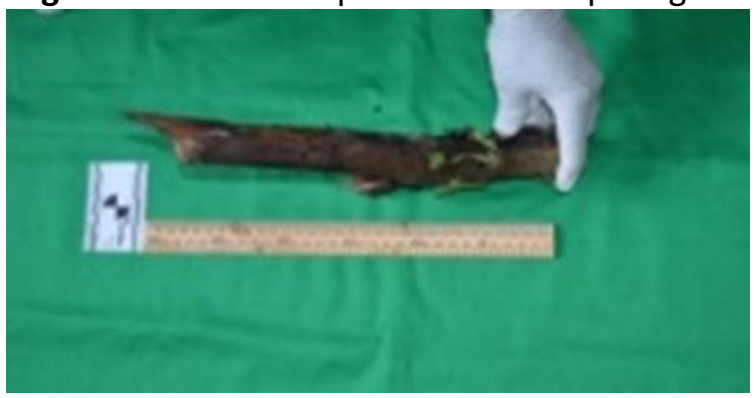

Figure 03. Shirt showing blood stains and defect

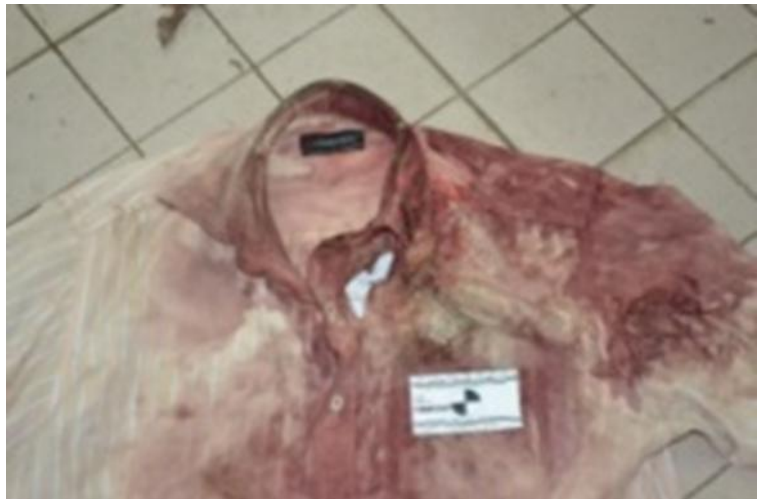

On dissection, underlying soft tissue contusion and contusion of sternocleidomastoid muscle was present. The wound was penetrating along the sternocleidomastoid muscle with a through and through perforation. Underlying complete transection of internal jugular vein with luminal thrombus formation was observed (Figure 05). The penetrating injury had gone through the thoracic inlet and terminated at the upper part of the left chest cavity causing a $400 \mathrm{ml}$ haemothorax. Small broken parts of the tree 
stump were found at the end of the wound. There were no injuries in the rest of the body. All organs were pale. Blood alcohol level was $116 \mathrm{mg}$ per dl. There was no natural disease pathology, which could have caused or contributed to the death.

Figure 04. Penetrating injury to neck

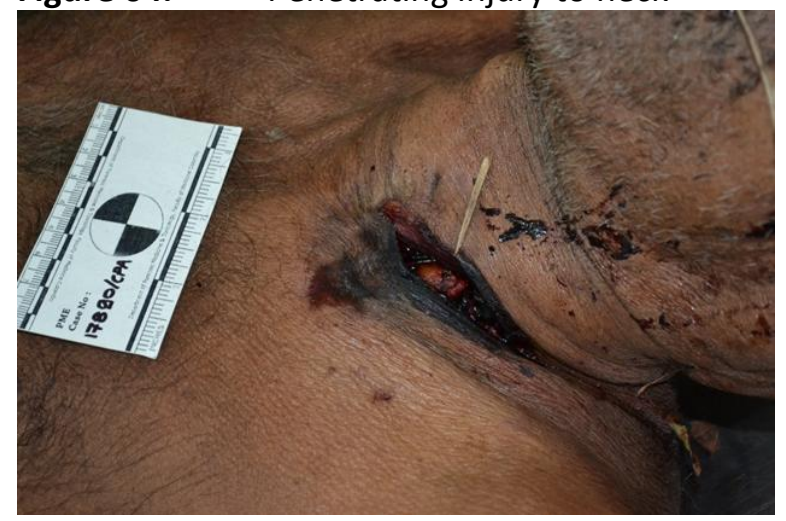

Figure 05. Transected Internal jugular vein showing thrombus

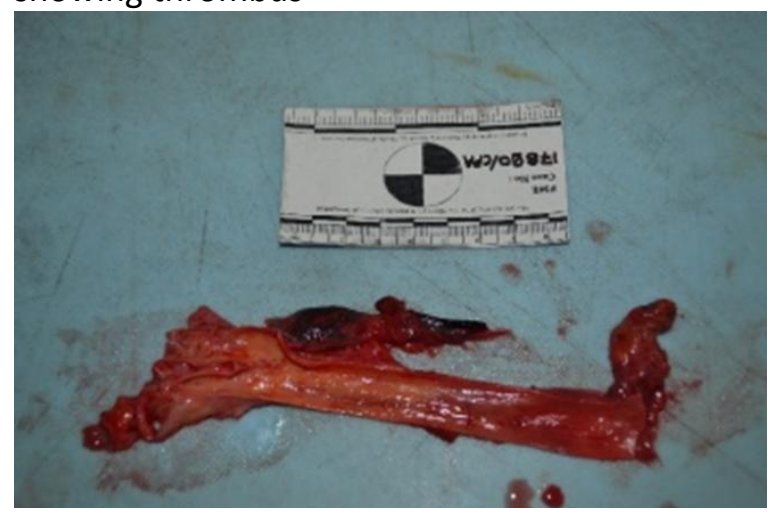

\section{Discussion}

Penetrating injury with a sharp pointed weapon like a knife would result in an injury with regular margins [04]. In this case, a blunt object with a tapering end would most likely be the offending agent due to the abraded, irregular margins of the injury and underlying soft tissue and muscle contusion. A visit to the scene was helpful in identifying the tree stump as the offending agent. The transection of the jugular vein would have led to severe bleeding as evidenced by the pool of blood at the scene. The $400 \mathrm{ml}$ haemothorax also would have contributed to death.

Penetrating neck and facial trauma represent approximately $5 \%$ to $10 \%$ of all trauma cases that present to the emergency department with a mortality of $1 \%$ to $6 \%$ [05,06]. Causes of penetrating head and neck injuries other than knife or gunshot wounds are rare, especially impalements [07]. They are most frequently seen in falls on construction sites onto iron bars found erect on the ground. Passengers who are ejected from automobiles onto wooden or metal post fences may also sustain these injuries. Impalement injuries also occur when a moving object penetrates a stationary body, usually occurring during traffic accidents where the patient can be impaled by a multitude of objects such as windshield wiper, metal guard rail, wooden rail fences and steel rods [08]. Impalement injuries to the neck as a result of falling upon a stump of a tree is an extremely rare event. One case is reported where a man fell off a tree and impaled on a pointing stick injuring the internal jugular vein [09]. However, there was no published report of a fatality due to impaling on a tree stump. If this victim died due to slow bleeding, there is a possibility that at the time of impalement he would have had a higher blood alcohol level than 116\% depending on the period of survival. This may have incapacitated him enough to be incapable of extricating himself from the tree stump, having impaled on it. The stump would have sunk deeper and deeper into the injury due to the weight of the body acting upon the neck.

In similar incidents, having sustained the injury if the victim walks away a significant distance, and the stump is not discovered at the scene, there can be strong suspicion of foul play. Therefore, when such penetrating injuries due to blunt pointed objects are detected, the scene should be meticulously searched for an offending agent before presuming that a crime has taken place.

Public awareness regarding the safe chopping of trees and effective covering of such redundant stumps is important especially as 
children can be subjected to the same circumstances while playing. Steel bars at construction sites should be guarded to eliminate this common construction site accident. Special caps which are designed to afford protection may be used to prevent such hazards of impalement. [2]

\section{References}

1. Missliwetz J, Fatal impalement injuries after falls at construction sites. Am J Forens Med Path 1995; 16 (1):81-3

2. OSHA construction e tool, Occupational Safety and Health administration, United states Department of Labour. Available at https://www.osha.gov/.../c...accessed on 10.01.2015/Occupational Safety and Health Administration

3. Watson J M, Goldstein L J, Golf club shaft impalement: case report of a zone III neck injury. Journal of Trauma.1996; 41(6):1036-8

4. Knight B, Saukko P. Knight's Forensic Pathology, 3rd edition, London, Arnold, 2004

5. Thal E R, Meyer D M. Penetrating neck trauma. Curr Probl Surg. 1992; 29(11): 55
6. Bostwick J III, Schneider WJ, Jurkiewicz MJ, et al. Penetrating injuries of the face and neck. South Med J. 1976;69:550 -553

7. Markey JC, Hines JL, Nance FC. Penetrating neck wounds: a review of 218 cases. Am Surg. 1975;41:77-83

8. Hyde MR, Schmidt CA, Jacobson JG, et al. Impalement injuries to the thorax as a result of motor vehicle accidents. Ann Thorac Surg. 1987;43:189-190

9. Wijeyaratne SM, Weerasinghe C, Cassim MRN. Blunt carotid injury from a penetrating stick: an unexpected injury. BMJ Case Reports 2010;2010:bcr0120102677. doi:10.1136/bcr.01.2010.2677.

\section{Acknowledgments:}

Authors wish to acknowledge the services of Mr. KMK Weerasuriya for obtaining the Photographs for this publication.

\section{Contribution of authors:}

Performing the autopsy - PA

Writing the manuscript - PA, JP

Revising manuscript - JP, RF 